\title{
Efficient visual search without top-down or bottom-up guidance
}

\author{
DELIANG WANG \\ Ohio State University, Columbus, Ohio \\ ARNI KRISTJANSSON \\ University College London, London, England \\ and \\ KEN NAKAYAMA \\ Harvard University, Cambridge, Massachusetts
}

\begin{abstract}
Two types of mechanisms have dominated theoretical accounts of efficient visual search. The first are bottom-up processes related to the characteristics of retinotopic feature maps. The second are topdown mechanisms related to feature selection. To expose the potential involvement of other mechanisms, we introduce a new search paradigm whereby a target is defined only in a context-dependent manner by multiple conjunctions of feature dimensions. Because targets in a multiconjunction task cannot be distinguished from distractors either by bottom-up guidance or top-down guidance, current theories of visual search predict inefficient search. While inefficient search does occur for the multiple conjunctions of orientation with color or luminance, we find efficient search for multiple conjunctions of luminance/size, luminance/shape, and luminance/topology. We also show that repeated presentations of either targets or a set of distractors result in much faster performance and that bottom-up feature extraction and top-down selection cannot account for efficient search on their own. In light of this, we discuss the possible role of perceptual organization in visual search. Furthermore, multiconjunction search could provide a new method for investigating perceptual grouping in visual search.
\end{abstract}

The feature integration theory of Treisman and Gelade (1980), proposed more than 20 years ago, continues to dominate the study of visual attention (Palmer, 1999; Pashler, 1998). According to this theory, the visual system first analyzes a scene in parallel by separate retinotopic feature maps. Focal attention then integrates analyses in different feature maps to produce a coherent perceptual object, and perceiving multiple objects in a scene requires a sequential shift of attention from one location to another. The theory has received support from extensive visual search experiments; for example, it successfully explains efficient search for targets that can be distinguished from distractors within a single-feature dimension, such as a red item in a field of green distractors. By efficient search, we mean that the slope of reaction time (RT) with respect to the set size (number of items) in a

Part of this work was undertaken while D.W. was a visiting scholar in the Harvard Vision Sciences Laboratory. Preliminary versions were presented in OPAM 1999 (Kristjansson, Wang, \& Nakayama, 1999) and ARVO 2000 (Nakayama, Kristjansson, \& Wang, 2000). We thank Jeremy Wolfe for comments on an earlier draft. D.W. was supported in part by an ONR YIP award, a National Science Foundation grant, and an AFOSR grant. A.K. was supported by a Fulbright graduate award, and A.K. and K.N. were supported in part by an AFOSR grant. Correspondence concerning this article should be addressed to D. Wang, Center for Cognitive Science and Department of Computer Science and Engineering, Ohio State University, 2015 Neil Ave., Columbus, OH 43210 (e-mail: dwang@cse.ohio-state.edu). display is small (e.g., $5 \mathrm{msec} /$ item) when the target is present, and inefficient search corresponds to a substantial search slope, say $20 \mathrm{msec} /$ item. A major prediction of the theory concerns conjunction search: the search for a target that is defined by a conjunction of features, say a red vertical target in a field of red horizontal and green vertical distractors. According to the theory, conjunction search requires that the observer attend serially to individual items; thus, it should be inefficient because the target can be distinguished from distractors only by examining multiple features simultaneously. In many cases, experimental results indeed confirm the predictions of the theory.

However, a number of experiments have shown that conjunction search can also be relatively efficient (under $10 \mathrm{msec} /$ item). For example, it has been shown that conjunctions of depth plane and color or motion produce very efficient search with a flat search slope (Nakayama \& Silverman, 1986), and even a conjunction of color and orientation can produce efficient search (Wolfe, Cave, \& Franzel, 1989). These results are in conflict with the feature integration theory and have led to modern revisions of the theory by Treisman and Sato (1990) and others. The so-called attentional engagement theory (Duncan \& Humphreys, 1992) consists of three components: a parallel description of individual items in a scene, matching between individual descriptions with a target description, and finally, weight linkage that serves to spread suppres- 
sion, caused by a mismatch, between like items. The attentional engagement theory is motivated by the observation that search performance depends on how similar distractors are to each other and how dissimilar a target is from distractors (Duncan \& Humphreys, 1989). Perhaps the most notable revision is Wolfe's guided search model (Wolfe, 1994; Wolfe et al., 1989), which hypothesizes two stages of processing, the first of which, consisting of parallel feature maps, is very similar to that of the feature integration theory. The main difference lies in the second stage, where the serial deployment of attention is guided by combined bottom-up and top-down activation. Bottomup activation is computed as the difference between an item and others in a local neighborhood, or in general by local spatial filters. Top-down activation results from selectively triggering the feature dimensions that characterize a predefined target. Thus, search for a conjunctively defined red vertical item, for instance, benefits from simultaneous top-down activation of a color map and an orientation map, which can explain efficient search performance. The guided search model not only avoids the problem of efficient conjunction search in the feature integration theory but also makes underlying mechanisms explicit, thus allowing for successful computer simulations of a range of empirical data (Wolfe, 1994, 1998).

Do bottom-up feature extraction and top-down selection form a sufficient basis to account for efficient visual search? To address this question, we have developed a search paradigm that we call multiple conjunction search. This paradigm extends Treisman's conjunction task in a way that the target is not uniquely defined on its own but defined only with respect to other items on a particular display. In a typical experiment, items are varied along two dimensions, such as luminance (black or white) and topology (disk or annulus); note that a disk and an annulus differ only in the presence of a hole, hence the name topology (see Chen, 1982). Figure 1 shows such a display. A target is defined as an odd-luminant disk or an odd-luminant annulus. Thus, one of four possible targets may appear on any trial: a black annulus among white annuli and black disks (Figure 1A), a white annulus among black annuli and white disks (Figure 1B), a black disk among white disks and black annuli (Figure 1C), or a white disk among black disks and white annuli (Figure 1D). In each of the four scenarios, the target is defined as a unique conjunction of luminance and topology, hence a multiconjunction. As in typical conjunction search, the target appears randomly on $50 \%$ of the trials. Items are equally distributed in each dimension and are randomly placed on a computer screen. This layout should eliminate bottomup guidance, since, as in conjunction search tasks, local comparisons among items do not yield salient activation that can be used to guide visual attention (see Figure 1). More important, because every item in the display can,



A

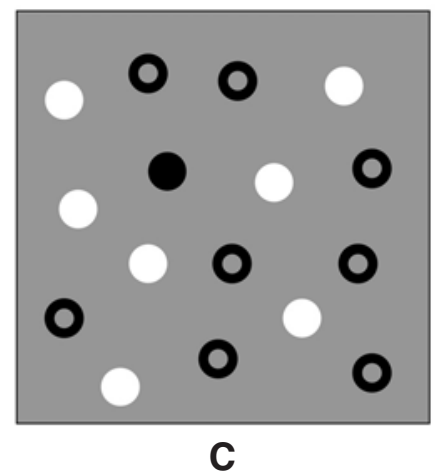



B



D

Figure 1. Four stimulus arrays illustrating different ways for a target to appear in multiconjunction search: (A) The target is the black annulus, (B) the target is the white annulus, (C) the target is the black disk, and (D) the target is the white disk. 
on its own, be either a target or a distractor, there should be no top-down guidance prior to each trial; in other words, top-down selection of a particular target provides no guidance because on any trial the item could also be, with equal probability, a distractor. In short, multiconjunction search should largely eliminate significant contributions from both bottom-up and top-down guidance from the search process.

For multiconjunction search tasks, standard theories of visual attention predict inefficient search with substantial search slopes because, lacking strong target saliency, the visual system would have to treat target and distractors alike and search through the display items one by one.

To preview the results, Experiment 1 shows that search for multiconjunctions of color and orientation is indeed inefficient. In contrast, Experiment 2 shows that three feature combinations - luminance/topology, luminance/ shape, and luminance/size - yield surprisingly efficient search. Experiment 3 further shows that a multiconjunction task combining two cases in Experiment 2 still yields efficient performance. In Experiment 4, we modified a multiconjunction task by simplifying the response procedure and found flat search slopes for both target-present and target-absent trials. In Experiment 5, we report that the multiconjunctions of luminance and orientation also lead to inefficient search, demonstrating that luminance alone cannot explain efficient performance in Experiment 2. Finally, Experiment 6 shows that priming for both targets and distractor sets leads to a dramatic reduction in RTs.

\section{EXPERIMENT 1 Inefficient Multiconjunction Search}

The main purpose of this experiment was to extend from standard conjunction search to multiconjunction search. The aim of this experiment was to test search performance for the multiconjunctions of color and orientation, the two feature dimensions frequently used in conjunction search tasks.

\section{Method}

Apparatus and Procedure. Stimuli were presented on a $75-\mathrm{Hz}$ monitor controlled by a Macintosh G3 computer. The display lay at the center of the monitor and was divided into $6 \times 6$ squares. The size of each square was $2.2^{\circ} \times 2.2^{\circ}$. Each stimulus item fit within a square and was centered on one of nine random positions within the square to produce some layout irregularity. The viewing distance was $57 \mathrm{~cm}$. On any given trial, the display array contained either 8 , 16,24 , or 32 items, and a target was present on $50 \%$ of the trials. Set size, locations of targets and distractors, and presence or absence of target varied randomly across trials.

The subjects were told to fixate a small cross at the center of the monitor and to respond to whether the target was present or absent by pressing two designated keys as quickly as possible, while maintaining a high degree of accuracy. The key corresponding to the target-present case was pressed by the right hand, and to the targetabsent case by the left hand. The display remained on the screen until the subject responded. A feedback tone was given to the subjects right after each trial to inform them whether their response was correct or not. Data associated with mistakes were discarded.
Each subject participated in a total of 330 trials. The first 30 trials were for practice, and the associated data were discarded. The remaining trials were divided into three blocks of 100 trials each. The subjects were allowed to take a short break between blocks.

Stimuli. Each stimulus item was a $1.1^{\circ} \times 0.28^{\circ}$ rectangular bar, oriented horizontally or vertically. The color of each bar was red $\left(12.7 \mathrm{~cd} / \mathrm{m}^{2}\right)$ or green $\left(11.8 \mathrm{~cd} / \mathrm{m}^{2}\right)$, and the background was black $\left(0.5 \mathrm{~cd} / \mathrm{m}^{2}\right)$. On a given display, except for a target item, half of the items were red and the other half were green; red items had one orientation (e.g., $0^{\circ}$ ), and green items had the other orientation (e.g., $90^{\circ}$ ). A target was defined as an odd-colored horizontal item or an oddcolored vertical item. Figure 1 illustrates the general relationship between a target and distractors in a typical multiconjunction task.

Subjects. Six students at Harvard University participated in the experiment. They all had normal or corrected-to-normal visual acuity and normal color vision. None of the subjects were aware of the purpose of the experiment, but they had substantial experience in taking part in psychological experiments.

\section{Results and Discussion}

Figure 2 shows the RT results for both target-present and target-absent cases. For the target-present case, the search slope is $24 \mathrm{msec} /$ item, whereas for the targetabsent case, the slope is $49.3 \mathrm{msec} /$ item. Such search performance is consistent with a so-called serial search, whereby attention presumably processes items sequentially until the target is identified. Moreover, performance is substantially worse than in standard conjunction search. For conjunction search with a very similar stimulus condition of color and orientation, FriedmanHill and Wolfe (1995) obtained a slope of $7 \mathrm{msec} /$ item for the target-present case and $8.9 \mathrm{msec} /$ item for the target-absent case. Comparable performances have been obtained by Wolfe et al. (1989), Treisman and Sato (1990), and also by us in a recent study (Kristjansson, Wang, \&



Figure 2. Mean reaction times plus $S E M$ with respect to set size in multiconjunction search of color and orientation. The search slope for the target-present case is $\mathbf{2 4} \mathbf{m s e c} / \mathrm{item}$, and that for the target-absent case is $\mathbf{4 9 . 3} \mathbf{~ m s e c / i t e m}$. 
Nakayama, 2002). Not only are slopes steeper, but intercepts are also significantly higher in multiconjunction search. A repeated measures analysis of variance (ANOVA) revealed significant effects of set size for both targetpresent responses $[F(3,15)=36.79, p<.01]$ and targetabsent responses $[F(3,15)=27.83, p<.01]$. Table 1 gives error rates in percentages for both target-present and target-absent cases and for all set sizes, as well as average error rate for each case and for each set size. The overall average error rate in this experiment is $6.1 \%$.

As explained earlier, standard search theories predict inefficient search for multiconjunction tasks because, lacking top-down or bottom-up guidance, attention would have to treat all items alike and search through them serially. This prediction is well supported by the data from this experiment.

Yet it is a logical possibility that some combinations of features might exist, whereby search could actually be efficient. Such cases would constitute counterexamples to the predictions of standard models of visual search. If they could be identified, they would argue for the existence of other potential mechanisms at work in visual search. Toward this end, the next experiment tested multiconjunction search under the following three stimulus conditions: luminance/topology, luminance/shape, and luminance/size.

\section{EXPERIMENT 2 Efficient Multiconjunction Search}

This set of experiments was designed to address the question of whether efficient search was at all possible in multiconjunction tasks. In the experiments, subjects searched for targets defined by the multiconjunctions of luminance and (1) topology, (2) shape, and (3) size, respectively.

\section{Method}

The apparatus and procedure part of this experiment was identical to that of Experiment 1.

Stimuli. As illustrated in Figure 1, each stimulus item was either black $\left(0.5 \mathrm{~cd} / \mathrm{m}^{2}\right)$ or white $\left(50.9 \mathrm{~cd} / \mathrm{m}^{2}\right)$, on a gray background $\left(33 \mathrm{~cd} / \mathrm{m}^{2}\right)$. Three stimulus conditions were tested. For the condition of luminance/topology (see Figure 1), disks and annuli were used. The diameter of a disk and the outer circle of an annulus was $0.82^{\circ}$, and that of the inner circle of an annulus was $0.43^{\circ}$. The target in this condition was defined as an odd-luminant disk or an oddluminant annulus. For the condition of luminance and shape, disks and horizontal bars were used. The disks are of the same size as those used in the first condition, and the size of a rectangular bar was $1.4^{\circ} \times 0.35^{\circ}$, so that the area was the same for the two shapes. The target in this condition was defined as an odd-luminant disk or an odd-luminant bar. For the condition of luminance and size,

Table 1

Error Rates (in Percentages) for Experiment 1

\begin{tabular}{lccccc}
\hline & \multicolumn{4}{c}{ Set Size } & \\
\cline { 2 - 5 } & 8 & 16 & 24 & 32 & Average \\
\hline Target present & 5.5 & 5.1 & 7.8 & 8.1 & 6.6 \\
Target absent & 3.1 & 4.3 & 4.9 & 6.3 & 4.7 \\
Average & 4.2 & 4.7 & 6.4 & 7.2 & 5.6 \\
\hline
\end{tabular}

squares of two sizes were used. The size of a large square was $0.82^{\circ} \times 0.82^{\circ}$, and that of a small square was $0.41^{\circ} \times 0.41^{\circ}$. The target in this condition was defined as an odd-luminant small square or an odd-luminant large square. The stimulus patterns used in the three conditions are illustrated in the inset of Figure 3.

Subjects. Six new subjects, recruited from the Ohio State University graduate student population, participated in the experiment. They all had normal or corrected-to-normal vision. They were naive to the purpose of the experiment and had never participated in any psychological experiment before. Each subject participated in all three stimulus conditions, for a total of 990 trials.

\section{Results}

Figure 3 shows the RT results for the three stimulus conditions. For the multiconjunctions of luminance/ topology, the search slope is near zero for the targetpresent case. For luminance/shape and luminance/size, the search slopes for target-present trials are 3.3 and $7.6 \mathrm{msec} /$ item, respectively. It is clear from Figure 3 that search is efficient for all three conditions, much more so than for the condition with orientation and color using an otherwise identical paradigm. Search is less efficient when the target is absent, but the pattern of target-absent performance in Figure 3 seems too efficient to support a serial rejection process. The target-absent case will be examined in Experiment 4, but we focus on the targetpresent case for now. A repeated measures ANOVA for the luminance/topology search reveals that the effect of set size is not significant for the target-present case $[F(3,15)=$ $0.98, p>.2]$, whereas it is significant for the targetabsent case $[F(3,15)=19.62, p<.01]$. For the luminance/ shape condition, the same $F$ ratios are $F(3,15)=1.93(p>$ $.05)$ and $F(3,15)=7.85(p<.01)$, respectively. For the luminance/size condition, they are $F(3,15)=3.98(p<$ $.05)$ and $F(3,15)=18.61(p<.01)$, respectively.

Table 2 gives percentage error rates for the three multiconjunction tasks for both target-present and target-absent cases and for all set sizes, along with average error rate for each case and set size. The overall average error rate is $8.1 \%$ for the topology task, $6.8 \%$ for the shape task, and $9.1 \%$ for the size task. Unlike the multiconjunctions of color and orientation, error rates are much higher for target-present cases than for target-absent cases. This issue will be discussed after Experiment 3 is reported below. We found little correlation between error rates and set sizes.

Search efficiencies in Figure 3 differ dramatically from those in Figure 2. Contradicting what is predicted by standard theories of visual search, for these stimulus conditions efficient search can take place without either top-down or bottom-up guidance. Before we discuss the implications of these surprising results, we will first report on a subsequent experiment.

\section{EXPERIMENT 3 Efficient Combined Multiconjunction Search}

Typical search tasks involve sessions of many consecutive trials where target and distractor identities do not vary from trial to trial. This experimental protocol leaves 




Figure 3. Mean reaction times plus $S E M$ with respect to set size in multiconjunction search for three different conditions: topology (discs and annuli), shape (bars and dises), and size (small and large squares). The three stimulus conditions are illustrated in the figure inset. Each item is either black or white on a gray background. For target-present cases, the slopes for the three conditions were $0.7 \mathrm{msec} /$ item, $3.3 \mathrm{msec}$ /item, and $7.6 \mathrm{msec}$ /item, respectively; for target-absent cases, the slopes were $9.1 \mathrm{msec} / \mathrm{item}, 12.6 \mathrm{msec} / \mathrm{item}$, and $16.1 \mathrm{msec} / \mathrm{item}$, respectively.

open the question of whether earlier trials have a facilitatory influence, or priming, on the present trial, because it has been demonstrated that between-trial priming can improve search performance considerably (Kristjansson et al., 2002; Maljkovic \& Nakayama, 1994). In the present situation, such priming is unlikely to be a significant factor, because one of four random conjunctions defines the target and the distractor set on each trial. To argue even more strongly against this possibility, we conducted an experiment that combined two conditions examined in Experiment 2: the luminance/topology condition and the luminance/size condition.

\section{Method}

The apparatus and procedure part of this experiment was identical to that of Experiment 1.

Stimuli. The stimulus patterns used in this experiment were the same as those used in the luminance/topology and the luminance/size conditions of Experiment 2. The target was defined as an oddluminant disk, an odd-luminant annulus, an odd-luminant small square, or an odd-luminant large square. As illustrated in Fig- ure 4A, distractors on a display are either small and large squares of different luminance, or disks and annuli of different luminance, that are consistent with the target item; for example, when the target is a disk or an annulus, the distractors are also disks and annuli.

Subjects. The subjects were the same 6 graduate students who participated in Experiment 2. They were naive to the purpose of the experiment.

\section{Results}

In this experiment, not even the dimensions along which features could vary were constant. On each trial, a target, when present, could be one of eight conjunctively defined items: four with disks and annuli, and another four with small and large squares. As with the targets, a distractor on a given trial could be one of the eight possible items. Figure 4B shows the RT results of this experiment. When a target was present, search was quite efficient, with a search slope less than $4 \mathrm{msec} /$ item. A repeated measures ANOVA showed that the effect of set size was significant for both the target-present case $[F(3,15)=$ $2.53, p<.05]$ and the target-absent case $[F(3,15)=$

Table 2

Error Rates (in Percentages) for the Three Conditions of Experiment 2

\begin{tabular}{|c|c|c|c|c|c|c|}
\hline & & \multicolumn{4}{|c|}{ Set Size } & \multirow[b]{2}{*}{ Average } \\
\hline & & 8 & 16 & 24 & 32 & \\
\hline Topology & $\begin{array}{l}\text { Target present } \\
\text { Target absent }\end{array}$ & $\begin{array}{r}15.6 \\
3.1\end{array}$ & $\begin{array}{r}15.9 \\
4.7\end{array}$ & $\begin{array}{r}14.7 \\
0.8\end{array}$ & $\begin{array}{r}16.7 \\
0.9\end{array}$ & $\begin{array}{r}15.7 \\
2.4\end{array}$ \\
\hline Shape & $\begin{array}{l}\text { Target present } \\
\text { Target absent }\end{array}$ & $\begin{array}{r}14.6 \\
5.2\end{array}$ & $\begin{array}{r}12.3 \\
3.1\end{array}$ & $\begin{array}{r}14.6 \\
4.6\end{array}$ & $\begin{array}{r}15.4 \\
3.6\end{array}$ & $\begin{array}{r}14.2 \\
4.1\end{array}$ \\
\hline Size & $\begin{array}{l}\text { Target present } \\
\text { Target absent }\end{array}$ & $\begin{array}{r}13.1 \\
2.7\end{array}$ & $\begin{array}{r}16.2 \\
2.3\end{array}$ & $\begin{array}{r}15.2 \\
2.1\end{array}$ & $\begin{array}{r}15.7 \\
2.7\end{array}$ & $\begin{array}{r}15.0 \\
2.4\end{array}$ \\
\hline Average & & 9.1 & 9.1 & 8.7 & 9.2 & 8.9 \\
\hline
\end{tabular}





Figure 4. Multiconjunction search for a combination of topology and size together with luminance. (A) A sequence of possible stimulus arrays illustrating multiconjunction search for a combination of disk/annulus and small/large squares. The target is absent in the third array and present in the other four arrays. (B) Mean reaction time plus $S E M$ with respect to set size. The slope is $3.8 \mathrm{msec} / \mathrm{item}$ for the target-present case and $15.5 \mathrm{msec} / \mathrm{item}$ for the target-absent case.

20.07, $p<.01]$, but we emphasize that the search slope for the target-present case is very shallow, indicating efficient search. Table 3 gives percentage error rates for both targetpresent and target-absent cases and for all set sizes as well as average error rates. The overall average error rate in this experiment was $7.7 \%$. The overall pattern of results for this multiconjunction task is similar to that in Experiment 2. This experiment shows that efficient search can occur with negligible repetition of either targets or distractor sets, thus reinforcing the results of Experiment 2.

\section{Discussion of Experiments 2 and 3}

These two experiments clearly demonstrate that efficient search can occur even when both top-down and bottom-up guidance are largely eliminated, and these experiments therefore contradict a basic prediction of the feature integration theory and its modern revisions that efficient search requires either top-down or bottom-up guidance. The multiconjunction search paradigm dimin- ishes the potential role of priming for either targets or distractors. In particular, Experiment 3 provides strong evidence for ruling out priming benefits as a cause for the observed efficient search performance. In other words, subjects appear to perform efficient search for targets on the basis of each individual display alone.

As mentioned earlier, the search slopes of response time versus set size are positive in the target-absent case in both Experiments 2 and 3. How an observer makes the no-target decision in a search task is not well understood

Table 3

Error Rates (in Percentages) for Experiment 3

\begin{tabular}{lrrrrr}
\hline & \multicolumn{4}{c}{ Set Size } & \\
\cline { 2 - 5 } & \multicolumn{1}{c}{8} & \multicolumn{1}{c}{16} & \multicolumn{1}{c}{24} & \multicolumn{1}{c}{32} & Average \\
\hline Target present & 13.8 & 15.0 & 14.8 & 13.5 & 14.3 \\
Target absent & 3.6 & 5.3 & 3.3 & 5.3 & 4.4 \\
Average & 8.7 & 10.1 & 9.1 & 9.4 & 9.4 \\
\hline
\end{tabular}


and probably involves a complex decision process. For instance, in the context of the guided search model, Chun and Wolfe (1996) introduced an activation threshold, below which no distractor is examined in the serial examination process of a display array. Therefore, some distractor items with very weak activation are eliminated from the search process altogether. They further assumed that this threshold is an adaptive one, increasing after correct responses (thus examining less of the display screen subsequently) and decreasing after incorrect responses (thus examining more of the display screen subsequently). Thus it is likely that decision criteria play a role in determining response time, and this may be part of the reason for the positive slopes.

Note also that there is a task asymmetry between the target-present case and the target-absent case in a typical search trial. The fact that an observer in a visual search experiment is instructed to look for a target gives the target-present case precedence over the target-absent case. The observer sets out to find a target on a display and, only when failing to do so, switches to decide on target absence. As specified in the Method section of Experiment 1 , the keys corresponding to these two cases require two different hands to press. This task asymmetry appears to be supported by a prominent trend of the error rates in Tables 2 and 3, in which a large majority of search errors occurs for the target-present case. That is, errors occur much more frequently when the target-absent key is pressed (but a target is present) than when the target-present key is pressed (but a target is absent). Given this fact, the Chun-Wolfe (1996) analysis on targetabsent trials suggests that subjects tend to be less certain when they do not see a target and thus examine more of the display before they press the target-absent key. It is possible that the amount of time spent on this extra screen examination is related to set sizes, yielding the upward slopes for target-absent cases in Experiments 2 and 3. Our next experiment attempts to minimize this task asymmetry by asking subjects to identify target presence and target absence separately.

\section{EXPERIMENT 4 Go/No-Go Search}

This experiment was designed to address the search asymmetry between target presence and target absence in the multiconjunctions of luminance and topology. Trials were divided into sessions, within each of which subjects were instructed to search for either target presence or target absence, but not both (a so-called go/no-go task). For example, within a target-presence session, subjects were instructed to respond if a target was present and do nothing otherwise and to do the same for target-absence sessions. This way, the search response is made simpler and the task asymmetry is removed.

\section{Method}

Apparatus and Procedure. As in previous experiments, stimuli were presented on a 75- Hz monitor of a Macintosh G3 computer.
In a go/no-go target-presence session, the subjects were instructed to respond with a keypress only if the target was present (on $50 \%$ of trials) but to "sit and wait" if the target was absent, in which case the trial ended after 1,500 msec. In a go/no-go target-absence session, the subjects were instructed to respond with a keypress only if the target was absent. The search task was the luminance/topology condition used in Experiment 2 (see Figure 1). As a control condition, the subjects also performed a standard multiconjunction search (indicating whether the target was present or absent by pressing corresponding keys with two hands).

Each subject participated in three sessions: one for multiconjunction, one for go/no-go target presence, and one for go/no-go target absence. The order of the sessions was randomly chosen for each subject. Each session consisted of 330 trials, and the first 30 trials were for practice purposes only.

Stimuli. The stimuli used in this experiment were the same as those used in the luminance/topology condition of Experiment 2. The target was defined as an odd-luminant disk or an odd-luminant annulus, as illustrated in Figure 1.

Subjects. Nine undergraduate students at Harvard University participated in this experiment for course credit. They all had normal or corrected-to-normal vision and were naive to the purpose of the experiment.

\section{Results and Discussion}

Figure 5 shows the RT results of this experiment. For the multiconjunction search, the slope for the targetpresent case is $-1.1 \mathrm{msec} /$ item, and for the target-absent case, it is $8.3 \mathrm{msec} / \mathrm{item}$. This pattern of results is very similar to that reported in Experiment 2. The higher intercepts in Figure 5 likely reflect the fact that subjects who participated in this experiment were different from those who participated in Experiment 2.

In contrast, for the go/no-go task the search slopes for both target-present and target-absent trials were flat; for the target-present case it is $-1 \mathrm{msec} / \mathrm{item}$, and for the target-absent case it is $0.9 \mathrm{msec} / \mathrm{item}$. If we compare the go/no-go data with those of multiconjunction, the most significant difference lies in target-absent performance. In addition, there is a large drop of RT for the go/no-go procedure, which has a lower task demand; subjects did not need to switch between the two tasks with two different motor responses. A repeated measures ANOVA shows a significant effect of set size only for the targetabsent case in the multiconjunction search $[F(3,24)=$ $18.71, p<.01]$. The $F$ ratios for the other three conditions (both go/no-go tasks and the target-present case for the multiconjunction search) are all less than 1, with $p \mathrm{~s}>.2$.

Table 4 gives percentage error rates for the above two experimental conditions and average error rates. Again, error rates are provided for both target-present and targetabsent cases and for all set sizes. The overall average error rate for the multiconjunction task is $6.8 \%$, and it is $2.4 \%$ for the go/no-go task. Note that for the go/no-go task, there are no appreciable differences in error rates for the target-present and target-absent cases.

The results of this experiment demonstrate that when target-present and target-absent tasks are made simpler and more symmetrical with the go/no-go procedure, the pattern of RT results for both tasks follows the same trend: The time that subjects take to perform either task 


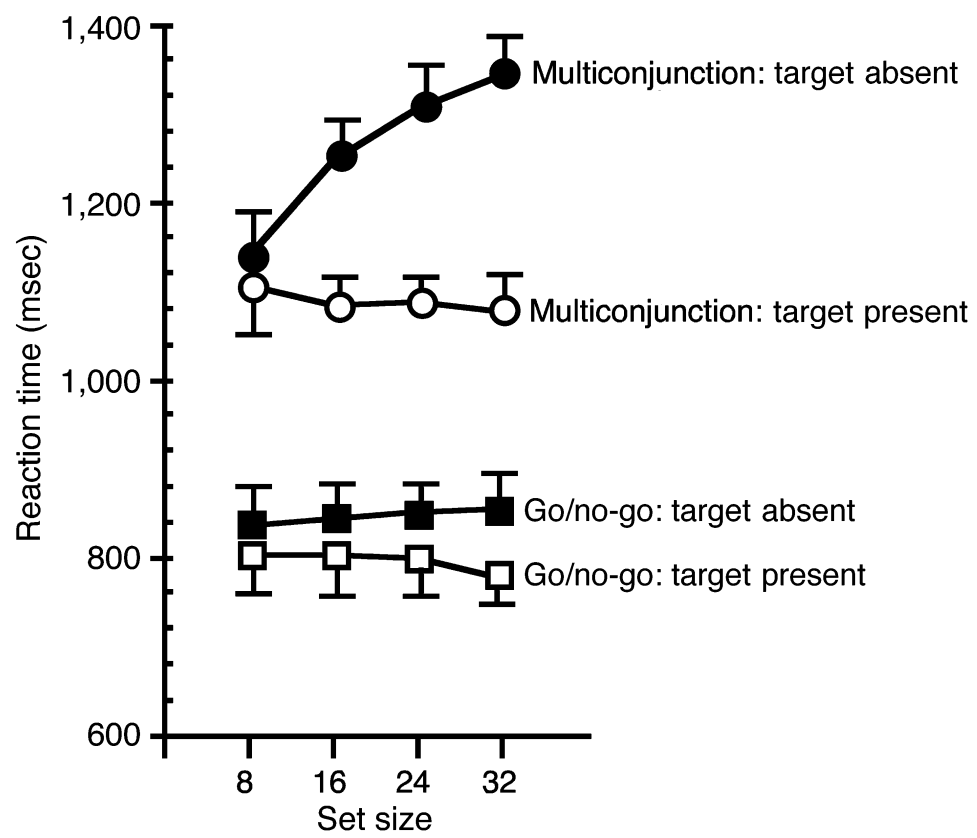

Figure 5. Mean reaction times plus $S E M$ with respect to set size in multiconjunction and go/no-go search tasks for the luminance/topology condition. For the multiconjunction task, the slope is $\mathbf{- 1 . 1} \mathrm{msec} / \mathrm{item}$ for the targetpresent case and $8.3 \mathrm{msec} /$ item for the target-absent case. For the go/no-go task, the slope is $-1 \mathrm{msec} / \mathrm{item}$ for the target-present case and $0.9 \mathrm{msec} / \mathrm{item}$ for the target-absent case.

does not change as the set size varies. Even when the stimulus array does not contain a target, subjects can process the array in parallel to perceive target absence. Because features that define distractor sets vary randomly across trials, the flat search slope for the targetabsent case poses a strong challenge to models of visual attention that rely on bottom-up and top-down activation. Such models, in the absence of top-down guidance and with items from two distractor sets spatially interleaved, predict a serial deployment of attention from one item to another, resulting in positive slopes of RT versus set size.

\section{EXPERIMENT 5 \\ Inefficient Search of Multiconjunctions of Luminance and Orientation}

So far, all efficient multiconjunction tasks have involved luminance as a stimulus feature. It has been shown that luminance can be more conducive to efficient search than can color. For example, Theeuwes and Kooi (1994) compared RT performance between luminance and color in a conjunction task involving the letters $\mathrm{X}$ and $\mathrm{O}$. They found efficient conjunction search with luminance but not with color. They attributed efficient search to dedicated neural pathways for luminance processing, suggesting a special status for luminance that is not shared by color. Thus, an important question in the present context is whether luminance alone is responsible for efficient multiconjunction search. To investigate this question, we performed an experiment on the multiconjunctions of luminance and orientation.

\section{Method}

The apparatus and procedure of this experiment were identical to those of Experiment 1.

Stimuli. Each stimulus item was a $1.1^{\circ} \times 0.28^{\circ}$ rectangular bar, oriented either horizontally or vertically. The size of the rectangular bar was identical to that in Experiment 1. Each item was either black $\left(0.5 \mathrm{~cd} / \mathrm{m}^{2}\right)$ or white $\left(50.9 \mathrm{~cd} / \mathrm{m}^{2}\right)$, and the background was gray $\left(33 \mathrm{~cd} / \mathrm{m}^{2}\right)$. This luminance layout was identical to that in Experiment 2. A target was defined as an odd-luminant horizontal item or an odd-luminant vertical item. See Figure 1 for the general relationship between a target and distractors in a typical multiconjunction search.

Subjects. Six new subjects, plus 1 who participated in Experiments 2 and 3, were tested. The subjects were recruited from the Ohio State University graduate student population. They all had normal or corrected-to-normal vision and were naive as to the purpose of the experiment. Five out of the 7 had never participated in any psychological experiment before.

\section{Results and Discussion}

Figure 6 shows the RT results for both target-present and target-absent cases. For the target-present case, the search slope is $23.8 \mathrm{msec} /$ item, whereas for the targetabsent case, the slope is $70 \mathrm{msec} /$ item. Table 5 gives percentage error rates for both target-present and targetabsent cases and for all set sizes together with average error rates. The overall average error rate for this task is 
Table 4

Error Rates (in Percentages) for Two Conditions of Experiment 4

\begin{tabular}{llrrrrr}
\hline & & \multicolumn{4}{c}{ Set Size } & \\
\cline { 3 - 6 } & & \multicolumn{1}{c}{16} & \multicolumn{1}{c}{24} & 32 & Average \\
\hline Multiconjunction & Target present & 9.4 & 10.1 & 11.6 & 9.5 & 10.2 \\
& Target absent & 5.1 & 4.1 & 4.6 & 3.7 & 4.4 \\
Go/no-go & Target present & 1.7 & 2.3 & 2.4 & 3.0 & 2.3 \\
& Target absent & 2.1 & 2.4 & 2.8 & 2.7 & 2.5 \\
Average & & 4.6 & 4.8 & 5.3 & 4.7 & 4.9 \\
\hline
\end{tabular}

$4.3 \%$. The pattern of the search performance is similar to that of Experiment 1, indicating serial deployment of attention. The higher intercepts in Figure 6 than in Figure 2 likely reflect the fact that a new subject group participated in this experiment. Furthermore, unlike those in Experiment 1, many of the subjects in this experiment had no experience with psychological experiments. There are significant effects of set size for both the targetpresent and target-absent response times $[F(3,18)=$ $10.16, p<.01$, and $F(3,18)=26.33, p<.01$, respectively], as revealed by a repeated measures ANOVA.

The results of this experiment demonstrate that luminance alone cannot explain efficient performance in multiconjunction search, although the use of luminance could facilitate some multiconjunction tasks. Such a facilitating role is supported by our informal observation that the search efficiency for the multiconjunctions of color and shape, involving disks and bars, is somewhat reduced in comparison with those of luminance and shape.

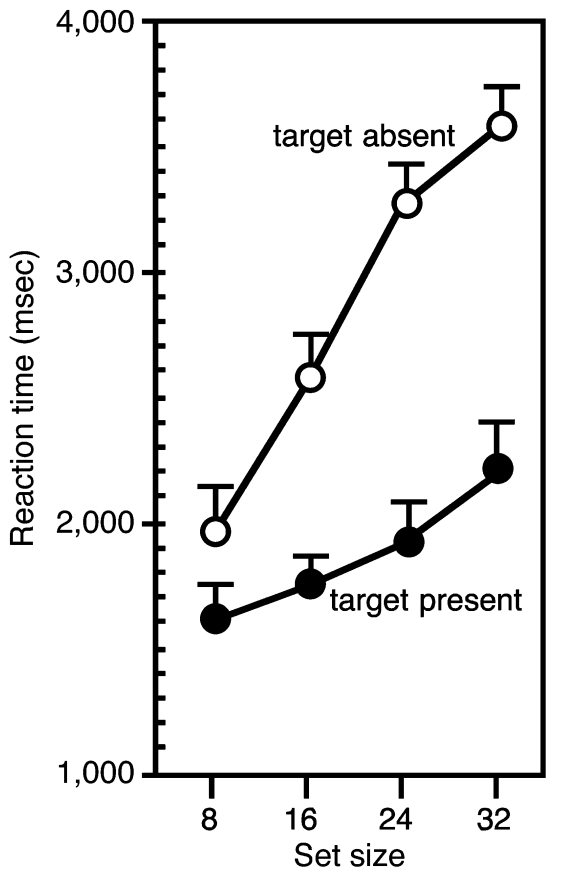

Figure 6. Mean reaction times plus $S E M$ with respect to set size in multiconjunction search of luminance and orientation. The slope for the target-present case is $\mathbf{2 3 . 8} \mathbf{~ m s e c / i t e m , ~ a n d ~ t h a t ~ f o r ~}$ the target-absent case is $70 \mathrm{msec} / \mathrm{item}$.
Taken together, these observations indicate that luminance and other stimulus features, such as topology and shape, combine to yield efficient multiconjunction search.

\section{EXPERIMENT 6 \\ Priming in Multiconjunction Search}

For efficient search based on individual features or a conjunction of features as in efficient conjunction search, RT intercepts typically range from 400 to $700 \mathrm{msec}$ for target-present cases (Friedman-Hill \& Wolfe, 1995; Nakayama \& Silverman, 1986; Theeuwes \& Kooi, 1994; Treisman \& Gelade, 1980; Treisman \& Sato, 1990; Wolfe et al., 1989). In comparison, RT intercepts in Figures 2 and 3 are higher than $850 \mathrm{msec}$. Multiconjunction search is more demanding than feature or conjunction search since there are four possible targets rather than one target, and subjects would be expected to take longer to respond. Experiment 4 demonstrates that a significant drop in RT intercepts results when the response procedure is simplified.

As alluded to earlier, one potential benefit not available in multiconjunction search is priming, which can occur when targets or distractors repeat their identities in consecutive trials. Typical experimental protocols employed in feature or conjunction search are subject to priming, which can significantly improve overall search performance (Kristjansson et al., 2002; Maljkovic \& Nakayama, 1994; Wolfe, Butcher, Lee, \& Hyle, 2003; see Kristjansson, in press, for a review). In Experiment 3, we showed that priming cannot explain efficient multiconjunction search. In this experiment, we modified the experimental procedure of a multiconjunction task in order to make it subject to potential priming and tested (1) whether priming would occur in the multiconjunction search paradigm and (2) whether priming, if it occurs, could bring down RT intercepts of multiconjunction search.

\section{Method}

Apparatus and Procedure. Unlike in regular multiconjunction search, where the target identity changes randomly between four possible ones from one trial to the next, in this experiment the target retained its identity for longer "streaks" than would be expected from a random choice of the target identity. This allowed us to document the effects of the repetition of the target (and distractor) identity across a number of trials - up to seven in a row in this experiment. The streaks were generated in the following way. The probability that the target on the current trial is the same as that on the previous trial is set to $1-n(0.1-0.01 n)$, where $n$ indicates how many 
Table 5

Error Rates (in Percentages) for Experiment 5

\begin{tabular}{lccccc}
\hline & \multicolumn{4}{c}{ Set Size } & \\
\cline { 2 - 5 } & 8 & 16 & 24 & 32 & Average \\
\hline Target present & 3.6 & 3.8 & 4.6 & 6.1 & 4.5 \\
Target absent & 3.3 & 4.0 & 4.1 & 5.1 & 4.1 \\
$\quad$ Average & 3.4 & 3.9 & 4.4 & 5.6 & 4.3 \\
\hline
\end{tabular}

times the same target has been repeated within a streak. Furthermore, the probability of repetition is set to the asymptote of .75 (when $n=5$ ) and to 0 when $n=7$, since the maximum streak length is set to 7. It is important to note that the target identity determines the corresponding two distractor sets (see Figure 1), whether a target actually occurs on the display or not. Hence, the target identity must be decided for each trial. With the repeating target identity, distractor identities repeat even when a target does not actually occur on a target-absent trial within a streak; in other words, $n$ is increased by 1 whether or not the target occurs on a given trial. The following example should further clarify how a streak was produced in the experiment. Assume that the target on the present trial (Trial 1) is a small square and the target on the previous trial is a different one. According to the probability formula, the target is set to the small square on Trial 2 (whether it is present or not) with a probability of .91. If the same small square is indeed assigned to be the target on the second trial, the same target repeats with a probability of .84 for Trial 3 , and so on. As a result, the target identity is never completely predictable from one trial to the next. But overall, the probability that the same target repeats from one trial to the next is considerably greater than .25 - the probability for a random selection from four possible targets as in regular multiconjunction search.

Each subject participated in a total of 830 trials. The first 30 trials were for practice only, and the associated data were not used in data analysis. The remaining 800 trials were divided into four blocks of 200 trials each, and the subjects were allowed to take a short break between blocks. More trials were needed for analyzing various streak lengths than in typical multiconjunction search-hence the greater number of trials.

The apparatus and procedure were otherwise the same as in Experiment 1 .

Stimuli. The stimulus patterns used in this experiment were the same as those for the luminance/size condition of Experiment 2. On each trial, the target could be one of the following: a small black square, a small white square, a large black square, or a large white square. Half of the distractors on each trial would differ from the target in size and the other half would differ from the target in luminance (either white or black).

Subjects. Six graduate students at Ohio State University participated in this experiment, 4 of whom had also participated in Experiments 2 and 3. They all had normal or corrected-to-normal vision and were naive to the purpose of the experiment.

\section{Results and Discussion}

We tested for potential priming for both targets and distractor sets by using streaks, where the same target and distractor sets in the multiconjunctions of luminance and size repeated for long stretches of consecutive trials (see the above Method section). If there were any priming benefits, we should have seen RTs drop as the number of repetitions increased. Figure 7A presents reaction times as a function of the position of a trial within a streak for both target and distractor repetitions. The data for the targetpresent case in Figure 7A show striking priming benefits. The negative slopes between RT and position in streak in- dicate that target repetitions speed up search by more than $60 \mathrm{msec}$ for each repetition. The benefits for the first few repetitions are more dramatic than for later ones. The pattern of improvement is similar for different set sizes, consistent with our finding that the RT slope for this multiconjunction task is small (see Figure 3). The right panel of Figure 7A presents data for distractor repetitions in a similar fashion. Again, there are strong priming effects for repetitions of distractor sets. Greater improvements occur for larger set sizes, which correlates with the data in Experiment 2 that RTs are higher for larger set sizes in target-absent trials. Strong priming effects for both targets and distractor sets suggest that priming in multiconjunction search works on the whole search array rather than being bound only to individual items. This observation extends our recent results on priming in conjunction and subset search paradigms (Kristjansson et al., 2002). Figure 7B provides the RT results averaged across different set sizes for both target-present and target-absent cases. Figure 7C plots the RTs with respect to set sizes separately for Trials 1-3 (squares) and Trials 5-7 (circles) within a streak, for both target-present and target-absent cases. Consistent with Figures 7A and 7B, significant priming occurs in both target-present and target-absent cases, with stronger effects for the target-present case where the intercept drops by approximately $200 \mathrm{msec}$. A repeated measures ANOVA showed a significant effect of streak length on RTs for both the target-present $[F(6,30)=$ $5.64, p<.01]$ and the target-absent $[F(6,30)=3.91, p<$ $.05]$ cases.

The results of this experiment demonstrate that priming can play a large role in visual search, which is often ignored in the literature (see Kristjansson et al., 2002, for further discussion).

Unlike in multiconjunction search, stimulus dimensions that define targets and distractors in feature search or conjunction search do not vary from trial to trial, and typically subjects are tested in blocks of 100 consecutive trials. That is, very long repetitions of target and distractor identities effectively result in very long streaks. Thus, it is reasonable to conclude that feature search and conjunction search enjoy maximal priming benefits, which could be larger than those shown in Figure 7 (about $400 \mathrm{msec}$ for the target-present case and $250 \mathrm{msec}$ for the target-absent case) for a limited range of streak lengths. Subtracting such benefits for the target-present case could bring down RTs of this task close to the typical range of 400-700 msec associated with efficient feature and conjunction search tasks. In other words, RTs in multiconjunction search may not be very different from those in regular search after priming effects are taken into consideration.

\section{GENERAL DISCUSSION}

The results of the experiments presented in this article demonstrate that efficient visual search, which is typically associated with the so-called popout of the target, 

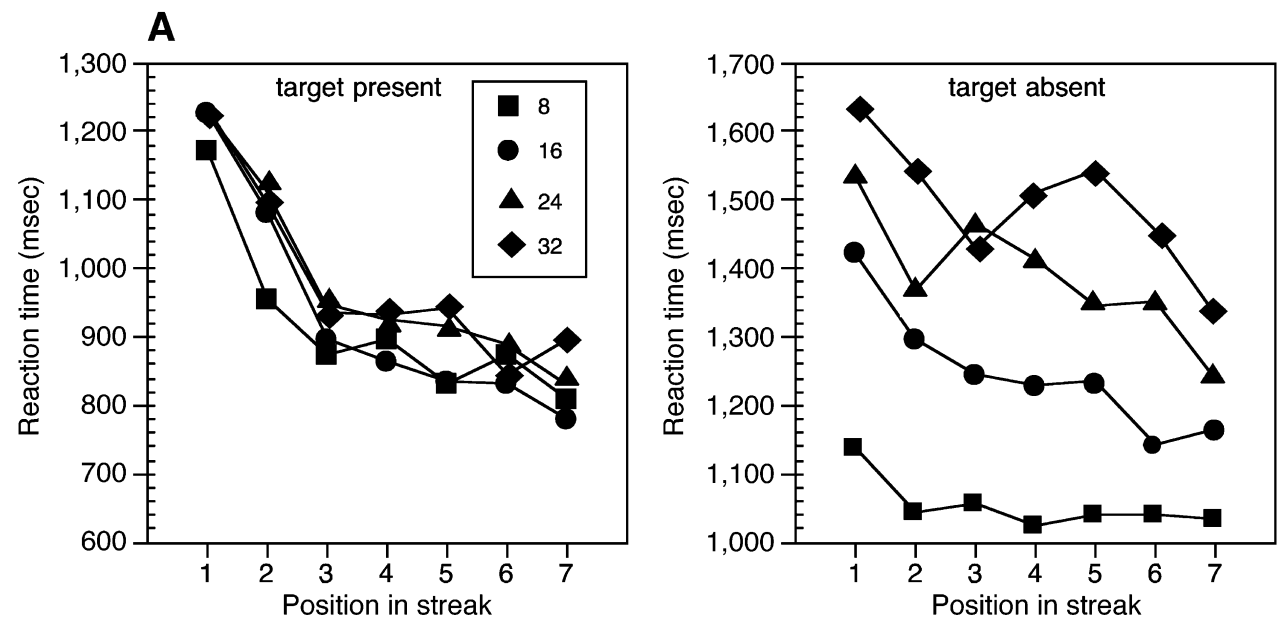

B

C
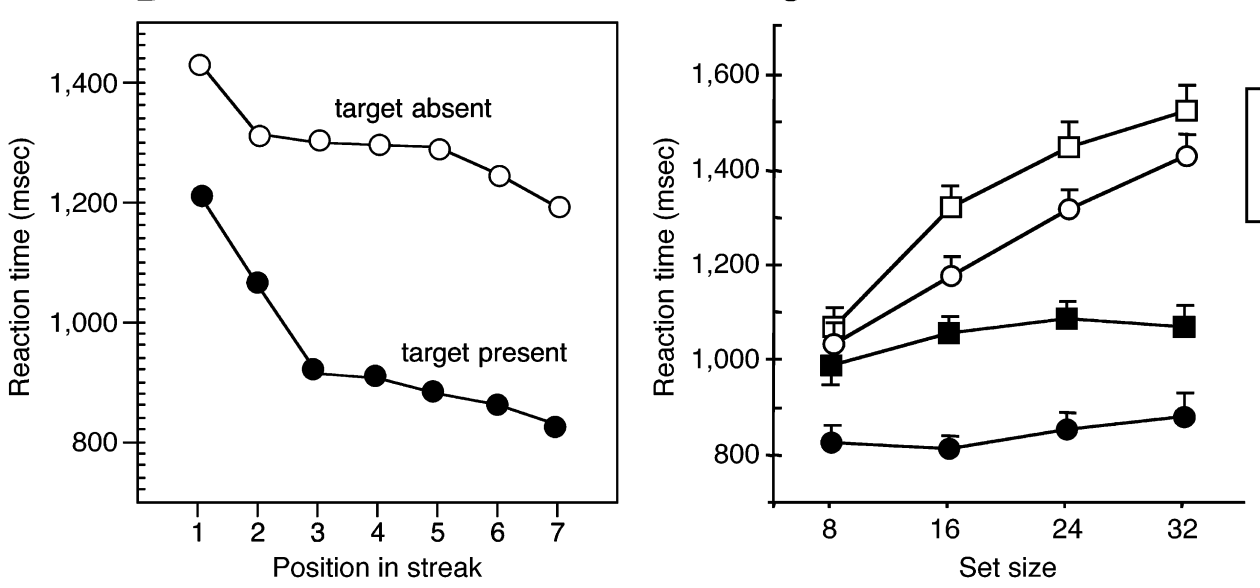

Figure 7. Priming effects for multiconjunctions of luminance and size. (A) Mean reaction times as a function of position in a streak. (B) Mean reaction times with respect to position in a streak, collapsed across all set sizes. (C) Mean reaction time with respect to set size for the first three trials (squares) and the last three trials (circles) within a streak. Both target-present and target-absent data are displayed.

can occur without significant contributions from either top-down or bottom-up guidance. Top-down guidance is provided by selective activation of feature dimensions that define a target, and bottom-up guidance results from local spatial filtering. The multiconjunction search paradigm introduced here largely eliminates both forms of guidance. Standard theories of visual attention rely on such guidance to explain efficient search, and thus cannot satisfactorily explain the results. If theoretical accounts of visual search are to explain our results, they need to posit explicit mechanisms that can explain the findings. Also, the demonstrated importance of priming of both targets and distractor sets shows how important priming can be for visual search performance, and this is consistent with our previous observation (Kristjansson et al., 2002) that a large portion of efficiency in conjunctive visual search can be accounted for by priming effects. Thus, priming also needs to be incorporated in visual search models.

\section{Search Efficiency}

In the visual search literature, the standard measure of search efficiency is the slope of RT with respect to set size. Another aspect of search efficiency, which is not usually discussed, is the intercept. Although we have clearly demonstrated efficient multiconjunction search in terms of the RT slope, RT intercepts in our multiconjunction search experiments are high in comparison with those in efficient feature or conjunction search. As we mentioned earlier, for target-present cases, RT intercepts in the multiconjunction search experiments are higher than $850 \mathrm{msec}$, whereas the intercepts range typically from 400 to $700 \mathrm{msec}$ in feature and conjunction search experiments. Two main reasons may explain the higher intercepts in multiconjunction search. First, the search procedure is more complex: There are four possible targets in multiconjunction search, and only one in feature and conjunction search. As a result, an observer likely takes longer to make a decision. From the data in 
Friedman-Hill and Wolfe (1995), for example, there is an intercept difference of about $100 \mathrm{msec}$ between two different feature search experiments for target-present cases: The intercept for searching for a red vertical target among red and green horizontal distractors is about $500 \mathrm{msec}$, whereas that for an oddly oriented target is about $600 \mathrm{msec}$. Their data also show that a subset search for a red, oddly oriented target produces an intercept that is about $150 \mathrm{msec}$ higher than that for the corresponding conjunction search for a red, vertical target.

Another likely reason for the higher intercepts in our experiments is within-trial priming. As discussed in Experiment 6 , both feature search and conjunction search enjoy maximal priming benefits because the target is fixed throughout an experiment; such benefits are absent in multiconjunction search due to the random change of targets. Experiment 6 demonstrates that priming in multiconjunction search can lower the intercept by $200 \mathrm{msec}$ or more. After these two factors are taken into account, the intercepts in multiconjunction search do not appear very surprising.

\section{A Possible Role for Perceptual Organization}

Besides top-down selection and bottom-up filtering, what perceptual processes might explain our experimental results? Our working hypothesis is that perceptual organization, which organizes visual elements into global perceptual units (Kanizsa, 1979; Koffka, 1935), could also play an important role in determining visual search performance. Two main reasons lead us to this suggestion. First, perceptual organization is a major process of visual perception (Palmer, 1999) and should intuitively be relevant to visual search and visual attention in general. Many previous studies have employed perceptual organization to account for a variety of phenomena in spatial vision, including texture segmentation (Beck, 1982; Julesz, 1981), object representation (Palmer \& Rock, 1994; see also Peterson \& Gibson, 1994, for the involvement of object recognition in perceptual organization), illusory conjunctions (Prinzmetal, 1995), perception of hierarchical patterns (Kimchi, 1998), and saccadic eye movements (Pomplun, Reingold, \& Shen, 2003). Indeed, Kahneman and Henik (1981) explicitly suggested that attention is allocated to preattentively organized perceptual units, and this notion is supported by visual search experiments (Kahneman \& Henik, 1981; Treisman, 1982). It is somewhat surprising that perceptual organization is largely missing from standard theories of visual attention (see also Pomplun et al., 2003). The second reason for suggesting that perceptual organization may explain our results is that this is consistent with a number of previous visual search studies indicating the involvement of perceptual grouping. Research using the so-called subset search paradigm shows that with explicit instruction to focus on a subset of items defined by a single feature dimension (e.g., red), the search for a conjunction target can be significantly facilitated (Bacon \& Egeth, 1997; Egeth, Virzi, \& Garbart, 1984). Duncan and Humphreys
(1989) showed that the use of a set of homogeneous distractors facilitates search performance, suggesting the role of similarity-based grouping of the distractor set. A study of conjunction search using form and motion shows that the addition of a set of dots moving together with either a target or distractors improves search performance significantly (Kingstone \& Bischof, 1999). Furthermore, it has been shown that three-dimensional layout plays a crucial role in facilitating visual search (Enns \& Rensink, 1990; He \& Nakayama, 1992; Ramachandran, 1988), and in each of these studies search becomes much more efficient if the distractor set can be seen as a group of elements lying on a smooth surface.

Because conventional search paradigms do not separate processes of bottom-up feature extraction, perceptual organization, and top-down feature selection (all of which likely play a role in a given search experiment), efficient search results tend to be open to different interpretations. Indeed, in the studies cited above, the potential involvement of top-down selection considerably complicates the picture; in particular, the prior specification of a fixed target permits not only top-down guidance but also allows for target/distractor priming, both of which can improve search efficiency. The multiconjunction search paradigm introduced in this article makes it possible to largely eliminate potential influences from top-down and bottom-up processes as well as priming benefits. Thus, our efficient search results provide stronger evidence for the involvement of perceptual organization. On the other hand, although we think that perceptual organization is the most plausible explanation, we recognize that the multiconjunction experiments do not manipulate perceptual grouping in a direct manner, which is needed to establish a more compelling argument.

How could a perceptual organization process account for our results? In such a process, items in a visual scene are organized into perceptual groups or segments, each of which would behave as a basic unit for attentional processing. A segment could be a single item or a group of items. More specifically, for the stimulus conditions examined in Experiments 2 and 3, items of like luminance and like topology, shape, or size could be grouped into single segments, and they would be separated from those with different stimulus values. Consider Figure 1, for example. Items may be grouped together on the basis of luminance and topology. When a target is present, perceptual organization would produce three segments, one of which would be the target itself. Attention would then process each segment serially (Kahneman \& Henik, 1981; Treisman, 1982) and should be able to identify the target efficiently because of the small number of groups that need to be examined. This account implies that perceptual grouping takes place on the basis of each display alone and not from priming built up from previous trials, which is consistent with our data.

Although perceptual organization is based on characteristics of local scene elements, we stress that it differs from bottom-up guidance (Itti \& Koch, 2001; Wolfe, 
1994). First, bottom-up guidance is generated by local computation via spatial filters. In contrast, perceptual organization yields global structures via a grouping-andsegmentation process that occurs across an entire display. Second, bottom-up guidance serves to guide attention to a particular item or location; perceptual organization, on the other hand, does not directly give rise to such guidance. Our explanation is consistent with neural network models that emphasize emergent grouping based on local connectivity between model neurons (Grossberg, Mingolla, \& Ross, 1994; Wang, 1999).

One might also interpret efficient multiconjunction search as two successive subset searches, say on the basis of luminance, in the following way. When searching for black items, white ones "fade" into the background, and subset search would result in a feature search, and vice versa (Egeth et al., 1984; Friedman-Hill \& Wolfe, 1995). We were aware of this explanation, so we asked our subjects right after Experiments 2 and 3 what strategy they employed to perform the search tasks, such as focusing on black items first and then white ones. Their answer was that they did not consciously employ a specific strategy, and the target, when present, simply "caught" their eyes. To more explicitly examine this possibility, in an informal study we had 3 subjects perform the same multiconjunction search of luminance and topology as in Experiment 2 (see Figure 1), but we instructed them to focus on black items first and then white items. That is, they were instructed to do two consecutive-subset searches if the target did not belong to the black subset. To encourage them to use this strategy, we had the subjects perform a standard subset search where the target was always a black item right before the consecutive-subset search. According to their reports, it was difficult to shift from one subset to the next during the trials, and they often ignored the instruction when a white target "occurred" to them (note that the task required them to respond as quickly as possible). Also, the consecutive-subset search interpretation would imply that multiconjunctions of luminance and any other feature lead to efficient search, which is in disagreement with the inefficient search observed in Experiment 5 for the multiconjunctions of luminance and orientation. Hence, we consider the interpretation unlikely for our results. On the other hand, we recognize that further investigation is needed in order to reach a definitive answer on this question.

Experiments 1 and 5 showed inefficient multiconjunction search involving orientation, and a perceptual organization account would imply that grouping between items of similar orientation is blocked by items of different orientation. There are indeed indications that orientation-based grouping is sensitive to spatial alignment between items. In particular, psychophysical evidence supports the notion of an association field that groups neighboring items of similar orientation that are aligned to form a curvilinear contour (Field, Hayes, \& Hess, 1993; Kovacs \& Julesz, 1993). Neurobiological findings also show that neurons in the primary visual cortex are interconnected by long-range horizontal connections, which link neurons with similar orientation responses (Gilbert \& Wiesel, 1989; Rockland \& Lund, 1983). Such alignment is not present in most visual search experiments (including ours). As such, it might provide an explanation as to why items of the same orientation do not seem to group across interleaved items of different orientation.

In an early study examining perceptual grouping and visual search, Treisman (1982) found evidence that attention shifts serially between groups rather than between individual items. In her experiments, groups were spatially well separated, and items of different groups were not interleaved, as were those in Figure 1. Thus, spatial proximity can be an effective grouping cue. In the experiments of Duncan and Humphreys (1989), distractors were arranged either homogeneously or heterogeneously. In their homogeneous conditions, which produced efficient search, distractors can again be grouped on the basis of proximity. Unlike these experiments, our experiments seem to suggest that grouping among similar items is possible in the presence of interleaving, dissimilar ones. Previous studies have shown that such grouping is possible in conjunction with depth, motion, or color, all of which have dedicated neural filters in the visual system. Our findings in Experiments 2 and 3 are striking, since they show that a combination of luminance with other features, which do not seem to have dedicated neural filters, can also yield such grouping. It is thus hard to treat the findings as "preattentive" exceptions because of the existence of neural pathways.

The results of Experiment 4, which demonstrated that a simpler go/no-go procedure yields efficient search for target-present trials as well as for target-absent ones, are particularly revealing. The feature integration theory has never offered a clear explanation for efficient search on target-absent or blank trials. This predicament exists even for feature search. For example, Figure 8 shows a feature search experiment conducted by Friedman-Hill and Wolfe (1995), where subjects searched for an oddoriented bar in an array of homogeneously oriented bars with two different colors (red and green). For both targetpresent and target-absent trials, search performance is efficient with flat search slopes. With target presence, the results fit the feature integration theory well. Without a target, however, how do subjects perceive target absence efficiently? Note that prior to each trial, subjects have no idea about target orientation, and yet they can reject all the items in parallel! From a different perspective, perceptual organization based on orientation would readily explain the results for both target-present and target-absent cases. Likewise, the substantial priming in both target-present and target-absent trials shown in Figure 7 suggests that priming acts on the whole search array rather than on individual items (see also Kristjansson et al., 2002), which poses difficulty for activationbased models but is consistent with a perceptual organization account. 


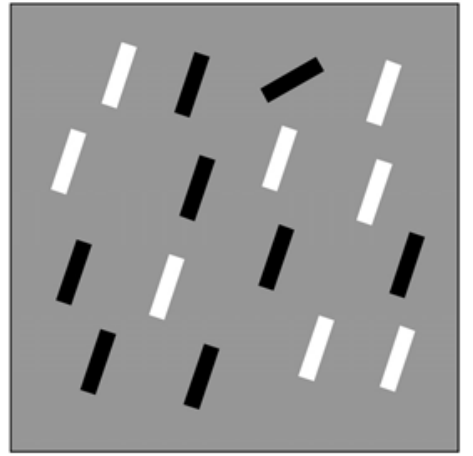

A



B

Figure 8. Orientation-based feature search. Items are either red (indicated by black) or green (indicated by white). (A) An example of the target-present case. The orientation of the target is different from that of other items. (B) An example of the target-absent case.

\section{Conclusion}

Using a new search paradigm, we have demonstrated that search can be efficient without explicit top-down or bottom-up guidance and that processes independent of spatial filtering and selection can determine whether search is efficient or laborious. An account consistent with the results from the six experiments described here is that visual attention serially examines perceptual groups rather than individual items (see also Kahneman \& Henik, 1981). It is possible that, under laboratory conditions, groups are the individual items themselves. But in many cases, a group consists of a set of items. Our results suggest that an adequate theory of visual attention needs to go beyond local filtering and top-down selection by incorporating perceptual grouping processes.

\section{REFERENCES}

BACON, W. F., \& EgETh, H. E. (1997). Goal-directed guidance of attention: Evidence from conjunctive visual search. Journal of Experimental Psychology: Human Perception \& Performance, 23, 948-961.

BECK, J. (1982). Textural segmentation. In J. Beck (Ed.), Organization and representation in perception (pp. 285-318). Hillsdale, NJ: Erlbaum.

CHEN, L. (1982). Topological structure in visual perception. Science, 218, 699-700.

CHUN, M. M., \& Wolfe, J. M. (1996). Just say no: How are visual searches terminated when there is no target present? Cognitive Psychology, 30, 39-78.

Duncan, J., \& Humphreys, G. W. (1989). Visual search and stimulus similarity. Psychological Review, 96, 433-458.

DunCAN, J., \& HumphreYs, G. W. (1992). Beyond the search surface: Visual search and attentional engagement. Journal of Experimental Psychology: Human Perception \& Performance, 18, 578-588.

Egeth, H. E., VirZI, R. A., \& Garbart, H. (1984). Searching for conjunctively defined targets. Journal of Experimental Psychology: Human Perception \& Performance, 10, 32-39.

EnNs, J. T., \& Rensink, R. A. (1990). Influence of scene-based properties on visual search. Science, 247, 721-723.

FiELD, D. J., HaYes, A., \& Hess, R. F. (1993). Contour integration by the human visual system: Evidence for a local "association field." $\mathrm{Vi}$ sion Research, 33, 173-193.

FRIEDMAN-HILL, S., \& WOLFE, J. M. (1995). Second-order parallel processing: Visual search for the odd item in a subset. Journal of Experimental Psychology: Human Perception \& Performance, 21, 531-551.
Gilbert, C. D., \& Wiesel, T. N. (1989). Columnar specificity of intrinsic horizontal and corticocortical connections in cat visual cortex. Journal of Neuroscience, 9, 2432-2442.

Grossberg, S., Mingolla, E., \& Ross, W. D. (1994). A neural theory of attentive visual search: Interactions of boundary, surface, spatial, and object representations. Psychological Review, 101, 470-489.

He, Z. J., \& NaKayama, K. (1992). Surfaces versus features in visual search. Nature, 359, 231-233.

IтTI, L., \& KoCH, C. (2001). Computational modelling of visual attention. Nature Reviews Neuroscience, 2, 194-203.

Julesz, B. (1981). Textons, the elements of texture perception, and their interactions. Nature, 290, 91-97.

Kahneman, D., \& HeNIK, A. (1981). Perceptual organization and attention. In M. Kubovy \& J. R. Pomerantz (Eds.), Perceptual organization (pp. 181-211). Hillsdale, NJ: Erlbaum.

Kanizsa, G. (1979). Organization in vision: Essays on gestalt perception. New York: Praeger.

KIMcHI, R. (1998). Uniform connectedness and grouping in the perceptual organization of hierarchical patterns. Journal of Experimental Psychology: Human Perception \& Performance, 24, 1105-1118.

KINGSTONE, A., \& BISCHOF, W. F. (1999). Perceptual grouping and motion coherence in visual search. Psychological Science, 10, 151-156.

KoFFKA, K. (1935). Principles of gestalt psychology. New York: Harcourt. Kovacs, I., \& JulEsz, B. (1993). A closed curve is much more than an incomplete one: Effect of closure in figure-ground segmentation. Proceedings of the National Academy of Sciences, 90, 7495-7497.

KRISTJANSSON, A. (in press). Rapid learning in attention shifts-a review. Visual Cognition.

Kristuansson, A., Wang, D. L., \& Nakayama, K. (1999). Grouping in visual search: Efficient search without top-down or bottom-up activation. In Proceedings of the 7th Annual Workshop on Object Perception and Memory (OPAM). Los Angeles.

Kristjansson, A., WAng, D., \& Nakayama, K. (2002). The role of priming in conjunctive visual search. Cognition, 85, 37-52.

Maljkovic, V., \& NakaYama, K. (1994). Priming of pop-out: I. Role of features. Memory \& Cognition, 22, 657-672.

Nakayama, K., Kristuansson, A., \& Wang, D. L. (2000). Efficient visual search not explained by local feature mechanisms or topdown guidance. Investigative Ophthalmology \& Vision Science, 41, S759.

Nakayama, K., \& Silverman, G. H. (1986). Serial and parallel processing of visual surface conjunctions. Nature, 320, 264-265.

PaLmer, S. E. (1999). Vision science. Cambridge, MA: MIT Press.

PALmer, S. [E.], \& Rock, I. (1994). Rethinking perceptual organization: The role of uniform connectedness. Psychonomic Bulletin \& Review, 1, 29-55.

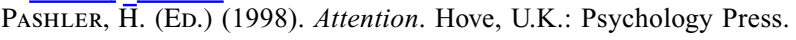


Peterson, M. A., \& Gibson, B. S. (1994). Must figure-ground organization precede object recognition? An assumption in peril. Psychological Science, 5, 253-259.

Pomplun, M., ReINGOLD, E. M., \& Shen, J. (2003). Area activation: A computational model of saccadic selectivity in visual search. Cognitive Science, 27, 299-312.

PRINZMETAL, W. (1995). Visual feature integration in a world of objects. Current Directions in Psychological Science, 4, 90-94.

RAMACHANDRAN, V. S. (1988). Perception of shape from shading. $\mathrm{Na}$ ture, 331, 163-165.

RoCKLAND, K. S., \& LuND, J. S. (1983). Intrinsic laminar lattice connections in primate visual cortex. Journal of Comparative Neurology, 216, 303-318.

Theeuwes, J., \& KoOI, F. L. (1994). Parallel search for a conjunction of contrast polarity and shape. Vision Research, 34, 3013-3016.

Treisman, A. (1982). Perceptual grouping and attention in visual search for features and for objects. Journal of Experimental Psychology: Human Perception \& Performance, 8, 194-214.

Treisman, A., \& Gelade, G. (1980). A feature-integration theory of attention. Cognitive Psychology, 12, 97-136.

Treisman, A., \& Sato, S. (1990). Conjunction search revisited. Jour- nal of Experimental Psychology: Human Perception \& Performance, 16, 459-478.

WANG, D. L. (1999). Object selection based on oscillatory correlation. Neural Networks, 12, 579-592.

WOLFe, J. M. (1994). Guided Search 2.0: A revised model of visual search. Psychonomic Bulletin \& Review, 1, 202-238.

Wolfe, J. M. (1998). Visual search. In H. Pashler (Ed.), Attention (pp. 13-73). Hove, U.K.: Psychology Press.

Wolfe, J. M., Butcher, S. J., Lee, C., \& Hyle, M. (2003). Changing your mind: On the contributions of top-down and bottom-up guidance in visual search for feature singletons. Journal of Experimental Psychology: Human Perception \& Performance, 29, 483-502.

Wolfe, J. M., CaVe, K. R., \& Franzel, S. L. (1989). Guided search: An alternative to the feature integration model for visual search. Journal of Experimental Psychology: Human Perception \& Performance, 15, 419-433.

(Manuscript received March 4, 2003;

revision accepted for publication May 11, 2004.) 